\title{
Transport Properties of Some Superconducting Multilayers
}

\author{
H. C. Yang ${ }^{a}$, L. M. Wang ${ }^{b}$, B. T. Su', H. H. Sung ${ }^{b}$ and H. E. Horng ${ }^{c}$ \\ ${ }^{a}$ Department of Physics, National Taiwan University, Taipei 106, Taiwan, R. O. C. \\ ${ }^{b}$ Department of Electrical Engineering, Da-Yeh University, Chang-Hwa 515, Taiwan, R. O. C. \\ ${ }^{\mathrm{c}}$ Department of Physics, National Taiwan Normal University, Taipei 117, Taiwan, R. O. C.
}

Transport properties of $\mathrm{YBa}_{2} \mathrm{Cu}_{3} \mathrm{O}_{\mathrm{y}} / \mathrm{Nd}_{0.7} \mathrm{Sr}_{0.3} \mathrm{MnO}_{3}$ (YBCO/NSMO) multilayers and $\mathrm{YBa}_{2} \mathrm{Ca}_{3} \mathrm{O}_{\mathrm{y}} / \mathrm{PrBa}_{2} \mathrm{Cu}_{3} \mathrm{O}_{\mathrm{y}}$ (YBCO/PBCO) superlattices were measured to characterize the activation energy and the upper critical field. The activation energy of the superconducting multilayers decreases as the thickness of the NSMO layer or the PBCO layer becomes thicker and the superconducting YBCO layer gets thinner. Temperature-dependence of upper critical fields shows a crossover from three-dimensional (3D) to two-dimensional (2D) behavior in YBCO/NSMO multilayers as the thickness of the YBCO layer is decreased. The results are discussed.

\section{INTRODUCTION}

Artificially grown superconducting (S) and ferromagnetic $(F)$ multilayers $[1,2]$ are of interest to study the superconductivity and magnetism. Particular interest in $\mathrm{S} / \mathrm{F}$ systems was renewed by the discovery of the divalence doped $\mathrm{R}_{1-\mathrm{x}} \mathrm{A}_{\mathrm{x}} \mathrm{MnO}_{3}$ compounds (where $\mathrm{R}=\mathrm{La}, \mathrm{Nd}, \mathrm{Pr}$, or $\mathrm{Sm}$, is some of the rare earth elements, and $\mathrm{A}=\mathrm{Sr}, \mathrm{Ca}, \mathrm{Ba}$, and $\mathrm{Pb}$ ), which exhibit "colossal" magnetoresistance [3]. In recent studies $[4,5]$, the fabrication of $\mathrm{Nd}_{0.7} \mathrm{Sr}_{0.3} \mathrm{MnO}_{3} / \mathrm{YBa}_{2} \mathrm{Cu}_{3} \mathrm{O}_{7} \quad$ (NSMO/YBCO) heterostructures has been reported also. On the other hand, superconducting properties of $\mathrm{YBa}_{2} \mathrm{Ca}_{3} \mathrm{O}_{\mathrm{y}} / \mathrm{PrBa}_{2} \mathrm{Cu}_{3} \mathrm{O}_{\mathrm{y}} \quad$ (YBCO/PBCO) superlattices with a stack of superconducting and semiconducting (s) layers have been studied widely [6]. In this paper, we report the transport characteristics in magnetic fields of YBCO/NSMO multilayers and YBCO/PBCO superlattices to investigate the flux dynamics and the dimensionality of vortex coupling by varying the vortex coupling between two superconducting layers separated by NSMO layers or PBCO layers.

\section{EXPERIMENT}

High quality YBCO, NSMO and PBCO thin films have been deposited onto $\mathrm{SrTiO}_{3}(100)$ substrates by an off-axis sputtering system. More detail study concerning the preparation of the multilayers was given elsewhere $[7,8]$. In the $\theta-2 \theta$ $\mathrm{x}$-ray scans, for the samples on $\mathrm{SrTiO}_{3}$ only the (00L) diffraction peaks of YBCO and PBCO were observed while the NSMO is oriented with the [110] direction perpendicular to the surface of the film. Transport properties were measured in fields parallel to the $c$-axis and the a-b plane up to $7 \mathrm{~T}$ with a current density of $\mathrm{J}=10^{4} \mathrm{~A} / \mathrm{cm}^{2}$.

\section{RESULTS AND DISCUSSION}

In the resistive measurements, the lower part of the resistive transition under magnetic fields can be described by the thermally activated flux motion [9]. It was observed that the activation energy of the superconducting multilayers decreases as the thickness of the NSMO layer or the PBCO layer become thicker and the superconducting YBCO layers get thinner. The activation energy of YBCO/NSMO multilayer is one order smaller than that obtained from YBCO/PBCO superlattices with about the same layer thickness. We observe a low pinning ability for YBCO/NSMO multilayers due to the pair-breaking effect by the spin polarization in the NSMO layers. The strong depinning due to the pair breaking mechanism is consistent with the observed depression of $T_{c}$ for YBCO/NSMO multilayers.

On other hand, it is known that the dimensionality can be examined from the temperature dependence of the upper critical fields [10]. For 3D superconductors, the temperature dependence of upper critical fields, $\mathrm{H}_{\mathrm{c} 2, \mathrm{c}}(\mathrm{T})$ and

\footnotetext{
*Supported by the National Science Council of R.O.C. under grant No. NSC 88-2112-M-212-002 and No. NSC 88-2112-M002-028.
} 
$\mathrm{H}_{\mathrm{c} 2 \mathrm{ab}}(\mathrm{T})$, along the $\mathrm{c}$-axis and the ab-plane can be described as

$$
H_{c 2, c}(T) \propto\left(1-T / T_{c}\right) \text { and } H_{C 2, a b}(T) \propto\left(1-T / T_{c}\right),
$$

While for 2D superconductors, the temperature dependence of $\mathrm{H}_{\mathrm{c} 2, \mathrm{c}}(\mathrm{T})$ and $\mathrm{H}_{\mathrm{c} 2, \mathrm{ab}}(\mathrm{T})$ is given by

$$
H_{c 2, c}(T) \propto\left(1-T / T_{c}\right) \text {, and } H_{C 2, a b}(T) \propto\left(1-T / T_{c}\right)^{1 / 2} \text {, }
$$

In Fig. 1 we show $\mathrm{H}_{\mathrm{c} 2, \mathrm{c}}(\mathrm{T})$ and $\mathrm{H}_{\mathrm{c} 2, \mathrm{ab}}(\mathrm{T})$ as a function of temperature for some $\mathrm{YBCO} / \mathrm{NSMO}$ multilayers and $\mathrm{YBCO} / \mathrm{PBCO}$ superlattices for the field parallel to the $c$-axis and the ab-plane. It was observed that $H_{c 2, c}(T)$ and $H_{c 2, a b}(T)$ show a linear behavior for the YBCO/NSMO $(240 \AA / 38.5 \AA) \times 4$ multilayer and the YBCO/PBCO $(120 \AA / 48 \AA) \times 8$ superlattice indicating the $3 \mathrm{D}$-like superconductivity. However, for the YBCO/NSMO $(120 \AA / 38.5 \AA) \times 8$ multilayer with a thinner YBCO layer, the $\mathrm{H}_{\mathrm{c} 2, \mathrm{c}}(\mathrm{T})$ shows a linear behavior whereas $\mathrm{H}_{\mathrm{c} 2, \mathrm{ab}}(\mathrm{T})$ was observed to be proportional to $\left(1-\mathrm{T} / \mathrm{T}_{\mathrm{c}}\right)^{1 / 2}$ as shown in Fig. 1(b). This temperature-dependence of $\mathrm{H}_{\mathrm{c} 2, \mathrm{c}}$ and $\mathrm{H}_{\mathrm{c} 2 \text {,ab }}$ suggest a crossover from a $3 \mathrm{D}$-like to a $2 \mathrm{D}$-like behavior in YBCO/NSMO multilayers as the thickness of the YBCO layer is decreased from $240 \AA$ to $120 \AA$. This
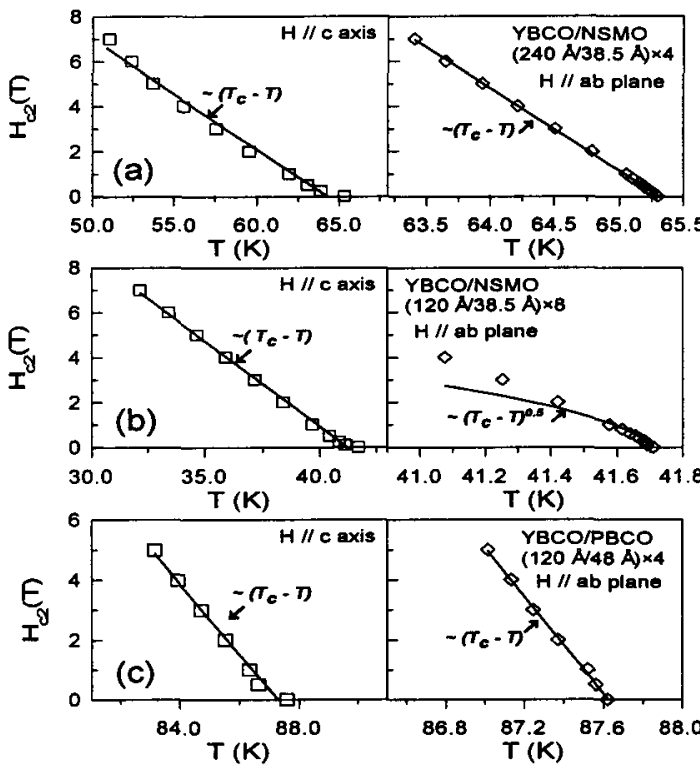

Figure 1. Temperature dependence of $\mathrm{H}_{\mathrm{c} 2, \mathrm{c}}$ and $\mathrm{H}_{\mathrm{c} 2, \mathrm{ab}}$ for (a) YBCO/NSMO $(240 \AA / 38.5 \AA) \times 4$, (b) $\mathrm{YBCO} / \mathrm{NSMO}(120 \AA / 38.5 \AA) \times 8$, and (c) YBCO/PBCO $(120 \AA / 48 \AA) \times 8$ multilayers.
2D-like behavior is attributed to the proximity induced ferromagnetic pair breaking in the decoupled YBCO layers.

\section{CONCLUSION}

The activation energy of the superconducting multilayers decreases as the thickness of the NSMO layer or the PBCO layer become thicker and the superconducting YBCO layers get thinner. Temperature-dependent upper critical fields suggest a crossover from 3D-like to 2D-like behavior in YBCO/NSMO multilayers as the thickness of the YBCO layer is decreased. The suppressions of $T_{c}$, pinning, and the 2D-like behavior of YBCO/NSMO multilayers are attributed to the proximity induced ferromagnetic pair breaking in the decoupled YBCO layers.

\section{REFERENCES}

1. K. Kawaguchi and M. Sohma, Phys. Rev. B 46, 14722 (1992).

2. L. Mieville, E. Keller, J.-M. Triscone and $\varnothing$. Fischer, Physica C 235-240, 725 (1994); L. Mieville, E. Keller, J.-M. Triscone, M. Decroux, $\varnothing$. Fischer and E. J. Williams, Phys. Rev. B 54, 9525 (1996).

3. S. Jin, T. H. Tiefel, M. McCormack, R. A. Fastmacht, R. Ramesh and L. H. Chen, Science 264, 413 (1994).

4. P. Przyslupski, S. Kolesnik, E. Dynowska, T. S. Koskiewicz and M. Sawicki, IEEE Trans. Appl. Supercon. 7, 2192 (1997).

5. Z. W. Dong, R. Ramesh, T. Venkatesan, Mark Johnson, Z. Y. Chen, S. P. Pai, V. Talyansky, R. P. Sharma, R. Shreekala, C. J. Lobb and R. L. Greene, Appl. Phys. Lett. 71, 1718 (1997).

6. H. C. Yang, L. M. Wang and H. E. Horng, Phys. Rev. B 59, 8956 (1999).

7. L. M. Wang, B.T. Su, H.C. Yang, and H.E. Horng, to be published in AIP conference series (EUCAS '99).

8. L. M. Wang, H. C. Yang and H. E. Horng, Phys. Rev. B 56, 6231 (1997).

9. P. W. Anderson and Y. B. Kim, Rev. Mod. Phys. 36, 39 (1964).

10. C. Uher, J. L. Cohn and I. K. Shuller, Phys, Rev. B 34, 4906 (1986). 